\title{
Helicobacter pylori induced transactivation of SRE and AP-1 through the ERK signalling pathway in gastric cancer cells
}

\author{
Y Mitsuno, H Yoshida, S Maeda, K Ogura, Y Hirata, T Kawabe, Y Shiratori, M Omata
}

\begin{abstract}
Background and aims-Helicobacter pylori infection induces expression of proinflammatory cytokines such as interleukin (IL)-8 and tumour necrosis factor $\alpha($ TNF- $\alpha)$ in gastric mucosa, and their genes have AP-1 binding sites in the promoter region. c-Fos is important for transactivation of AP-1 which has SRE in the promoter region. We conducted this study to confirm $H$ pylori induced transactivation of these binding sites.
\end{abstract}

Methods-Transactivation of SRE and AP-1 was evaluated in human gastric cancer cells TMK1 and MKN45 by luciferase reporter assay in transient transfection. We compared the effects of coculture with four $H$ pylori strains, a cag pathogenicity island (PAI) positive strain TN2, its isogenic vacA negative (TN2- $\Delta v a c A)$ or $c a g E$ negative (TN2- $\Delta c a g E$ ) mutants, and a cag PAI negative clinical isolate T68. Phosphorylation of ERK1/2, JNK, and c-Jun was measured by immunoblot, induction of IL-8 secretion by ELISA, and the effects of MEK by inhibitor U0126.

Results-Both SRE and AP-1 were transactivated by coculture with TN2. Although TN2- $\Delta v a c A$ induced comparable transactivation, TN2- $\Delta$ cagE and T68 showed decreased transactivation of SRE (65\% and $51 \%)$ and AP-1 (71\% and 54\%, respectively, of TN2). Heat killed TN2 or indirect contact using a permeable membrane inhibited transactivation. Levels of phosphorylated ERK1/2, JNK, and c-Jun were increased by coculture with TN2. MEK inhibitor U0126 reduced TN2 induced transactivation of SRE and AP1, as well as secretion of IL-8, by $83 \%, 87 \%$, and $53 \%$, respectively, of TN2.

Conclusions-Transactivation of SRE and AP-1, through ERK/MAPK and JNK/ SAPK cascades, respectively, was found in gastric cancer cells cocultured with $H$ pylori. Direct contact with viable bacteria possessing intact cag PAI is a prerequisite for the onset of intracellular signalling leading to AP-1 transactivation.

(Gut 2001;49:18-22)

Keywords: Helicobacter pylori; SRE; AP-1; cag pathogenicity island PAI; gastric cancer

Helicobacter pylori infects human gastric mucosa and induces chronic active gastritis. ${ }^{12} H$ pylori mediated inflammation is characterised by neutrophil infiltration mediated by interleukin (IL)-8 secretion from gastric epithelial cells. $^{3-5}$ Activation of transcriptional factors nuclear factor $\kappa \mathrm{B}(\mathrm{NF} \kappa \mathrm{B})$ and $\mathrm{AP}-1$ plays an important role in IL-8 induction. ${ }^{6}$ The cag pathogenicity island (cag PAI) of $H$ pylori, a cluster of approximately 30 genes, is a prerequisite, at least for induction of $\mathrm{NF \kappa B}$ activation. $^{78}$

AP-1 is a multipotential transcriptional factor with various cytokines and chemokines such as IL-2, IL-3, IL-4, IL-6, IL-8, and tumour necrosis factor $\alpha(\mathrm{TNF}-\alpha)$, with its binding site in their promoter region. ${ }^{9-12} \mathrm{AP}-1$ is formed either as a homodimer of c-Jun or as a heterodimer of c-Jun and c-Fos, with the latter form known to be more potent than the former. ${ }^{13}{ }^{14}$ While $H$ pylori mediated activation of the JNK/SAPK cascade leading to AP-1 transactivation was recently reported, ${ }^{15}$ the status of c-Fos in relation to $H$ pylori infection has not yet been elucidated. However, it is known that c-Fos expression is dependent on the ERK/MAPK cascade where activated Elk1 together with $\mathrm{SRF}^{1617}$ binds the SRE binding site in the promoter region of c-fos.

Thus we hypothesised that the AP-1 binding site is activated in $H$ pylori infection by the c-Jun/c-Fos heterodimer and sought to examine transactivation of SRE. In this study, we examined $H$ pylori mediated transactivation of both SRE and AP-1 directly by using a luciferase reporter assay, and analysed the ERK/MAPK cascade, a pathway upstream of SRE transactivation.

\section{Materials and methods}

BACTERIAL STRAINS

The TN2 strain, generously donated by $\mathrm{Dr}$ Nakao (Takeda Chemical Industries, Ltd, Osaka, Japan), was positive for two known virulence factors, CagA and VacA (vacuolating cytotoxin), and possessed intact cag PAI. ${ }^{18}$ Infection with this strain induced gastric cancer in Mongolian gerbils. ${ }^{19}$ The T68 strain, isolated from a Japanese patient at our institution, was negative for the above two virulence factors and lacked cag PAI. ${ }^{18}$ An isogenic cagE negative mutant (TN2- $\Delta c a g E$ ) was constructed by inserting a kanamycin resistant gene cassette into the cagE locus of cag PAI of $\mathrm{TN} 2$, as described in one of our previous papers. ${ }^{20}$ The isogenic vac $A$ negative mutant (TN2- $\Delta v a c A$ ) was constructed by disrupting

Abbreviations used in this paper: IL, interleukin;

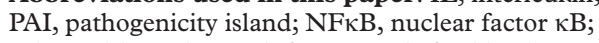
EGF, epidermal growth factor; FBS, fetal bovine serum. 
the $\operatorname{vac} A$ gene of TN2 which was confirmed by Southern hybridisation and vacuolation assay, as we described previously. ${ }^{21}$ These strains were cultured on Columbia agar with $5 \%$ (vol $/ \mathrm{vol}$ ) horse blood and Dent antibiotic supplement (Oxoid, Basingstoke, UK) at $37^{\circ} \mathrm{C}$ under microaerobic conditions (Campy-Pak Systems; BBL, Cockeysville, Maryland, USA). Heat killed $H$ pylori was prepared by treating the bacteria at $65^{\circ} \mathrm{C}$ for 60 minutes.

PLASMIDS

The reporter plasmids (Stratagene, La Jolla, California, USA) contained a Photinus pyralis luciferase gene driven by a basic promoter element (TATA) and seven AP-1 binding sites in pAP1-Luc, or by the basic promoter element and five SRE binding sites in pSRE-Luc. The efficacy of transfection was verified by cotransfection of a control plasmid (pRL-TK; Promega, Madison, Wisconsin, USA) containing the Renilla reniformis luciferase gene driven by the herpes simplex virus thymidine kinase.

REAGENTS

Recombinant human IL-1 $\beta$ was purchased from Upstate Biotechnology (Lake Placid, New York, USA); recombinant human epidermal growth factor (EGF) from Sigma (St Louis, Missouri, USA); polyclonal antibodies for phospho-p44/ p42 ERK1/2 (Thr202/Tyr204), p44/p42 ERK1/2, phospho-JNK/SAPK (Thr183/ Tyr185), JNK/SAPK, phospho-c-Jun (Ser63), and c-Jun from New England BioLabs, Inc. (Beverly, Massachusetts, USA); and MEK inhibitor U0126 from Promega.

HUMAN CELL LINES

Human gastric cancer cells TMK1 (a gift from Dr E Tahara, Hiroshima University School of Medicine, Hiroshima, Japan $)^{22}$ and MKN45 were maintained in RPMI 1640 (Sigma) supplemented with $10 \%$ fetal bovine serum (FBS) (Gibco/BRL), L-glutamine, penicillin G, and streptomycin. In coculture experiments, cancer cells $\left(4 \times 10^{5}\right.$ cells $\left./ \mathrm{ml}\right)$ and $H$ pylori $\left(10^{7}\right.$ colony forming units $/ \mathrm{ml}$ ) were cultured in RPMI 1640 without antibiotics and supplemented with $10 \%$ FBS. To assess the effects of direct contact, cancer cells and bacteria were separated by a membrane filter (Nunc Tissue Culture Inserts No 162138, Nunc, Roskilde, Denmark).

TRANSACTIVATION OF SRE AND AP-1 SITES

Transactivation of SRE and AP-1 was evaluated with the luciferase reporter assay. Approximately $4 \times 10^{5}$ cells were plated onto six well tissue culture plates (Iwaki Glass, Chiba, Japan) and transfected 24 hours later with the expression vector pSRE-Luc $(0.6 \mu \mathrm{g})$ or AP1-Luc $(0.6 \mu \mathrm{g})$ using Effectene transfection reagent (Quiagen, Hilden, Germany). Control vector pRL-TK $(0.01 \mu \mathrm{g})$ was added to each sample for standardisation of transfection efficacy. When pSRE-Luc reporter plasmids were transfected, the concentration of FBS in RPMI 1640 was reduced to $0.5 \%$ to minimise the effects of various factors in serum. After 24 hours, $H$ pylori $\left(10^{6}\right.$ colony forming units $\left./ \mathrm{ml}\right)$ was added. After another 20 hours of coculture, cells were harvested in phosphate buffered saline and lysed in a luciferase lysis buffer, and luciferase assays were carried out using the PicaGene dual seapansy system (Toyo Ink, Tokyo, Japan). Firefly luciferase activity and seapansy luciferase activity were measured as relative light units with a luminometer (Lumat LB9507; EG\&G Berthold, Bad Wildbad, Germany). Firefly luciferase activity was normalised for transfection efficiency based on seapansy luciferase activity. All assays were performed in four independent experiments.

U0126, a potent and specific MEK inhibitor of MEK1 and MEK2, ${ }^{23}$ was added to the culture medium at a final concentration of $10 \mu \mathrm{M}$, two hours before addition of $H$ pylori. The final concentration of dimethyl sulphoxide, used as a solvent, was $0.1 \%$ ( $\mathrm{vol} / \mathrm{vol}$ ), which did not affect the activity or activation of any protein kinase examined.

PHOSPHORYLATION OF ERK1/2, JNK, AND c-JUN After coculturing with $H$ pylori or stimulation by EGF or IL-1 $\beta$, cells were lysed in lysis buffer (50 mM Tris $\mathrm{HCl}$ buffer (pH 7.4) containing $1 \mathrm{mM}$ EGTA, $2 \mathrm{mM}$ dithiothreitol, $25 \mathrm{mM}$ sodium $\beta$-glycerophosphate, $0.1 \mathrm{mM}$ phenylmethylsulfonyl fluoride, and $10 \mu \mathrm{g} / \mathrm{ml}$ aprotinin) and centrifuged at $15000 \mathrm{~g}$ for $15 \mathrm{~min}$ utes. An aliquot of supernatant containing $10 \mu \mathrm{g}$ protein was applied on sodium dodecyl sulphate-polyacrylamide gel and transferred to a PVDF membrane (Amersham Pharmacia Biotech., Buckinghamshire, UK). The membrane was probed with an antibody to phosphorylated or total ERK1/2, JNK, or c-Jun at a dilution ratio of $1: 1000$ in Tris buffered salineTween. After washing, the membrane was incubated with horseradish peroxidase conjugated goat antirabbit immunoglobulin $G$ at a dilution ratio of 1:1000 in Tris buffered salineTween, and applied to the ECL detection assay (Amersham Pharmacia Biotech).

INTERLEUKIN-8 SECRETION

After TMK1 cells were cocultured with $H$ pylori for 20 hours, IL- 8 concentration in the culture supernatant was measured with an EIA (PerSeptive Diagnostics, Framingham, Massachusetts, USA) using a standard curve obtained from sequentially diluted recombinant IL-8 samples.

STATISTICS

Differences in means were analysed using the unpaired Student's $t$ test, and Dunnett's multiple comparison using SAS software version 6.12 (SAS Institute, Inc., Carey, North Carolina); $\mathrm{p}<0.05$ was considered significant.

\section{Results}

SRE TRANSACTIVATION BY H PYLORI

The luciferase assay using pSRE-Luc showed that coculture with TN2 strain enhanced SRE transactivation in TMK1 and MKN45 cells to $310 \%$ and $680 \%$ of responses in untreated cells, respectively (fig $1 \mathrm{~A}, \mathrm{~B}$ ). TN2- $\Delta v a c A$ showed comparable enhancement. However, T68, lacking cag PAI, reduced transactivation 
TMK1

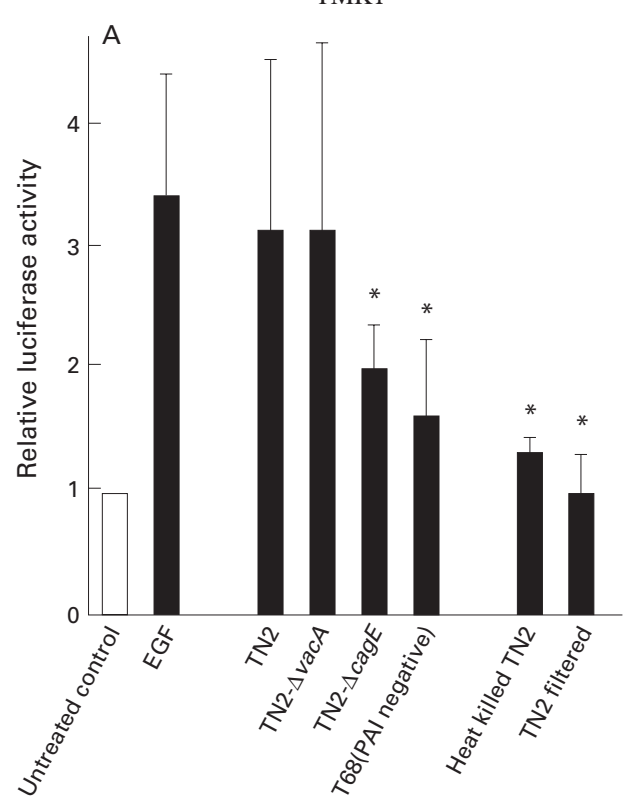

MKN45

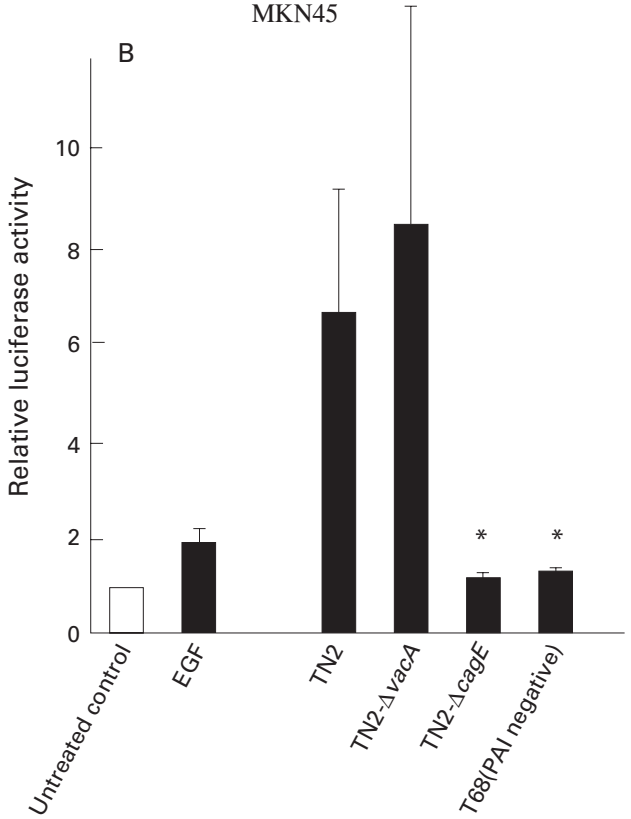

Figure 1 Transactivation of SRE by Helicobacter pylori. Transactivation of SRE in TMK1 (A) and MKN45 (B) cells induced by H pylori was measured by luciferase assay using pSRE-Luc. The TN2 strain was positive for two known virulence factors, CagA and VacA, and had intact cag pathogenicity island (PAI). The T68 strain, isolated from a Fapanese patient at our institution, was negative for the above two virulence factors and lacked cag PAI. An isogenic cagE negative mutant (TN2-AcagE) was constructed by inserting a kanamycin resistant gene cassette into the cagE locus of cag PAI of TN2. The isogenic vacA negative mutant (TN2-AvacA) was constructed by disrupting the vacA gene of TN2. Luciferase activity is presented as a fold induction relative to basal levels measured in untreated cells. Mean (SD) values of four independent experiments are shown. ${ }^{\star} p<0.05$ compared with TN2 by Dunnett's multiple comparison.

of $\mathrm{AP}-1$ to $52 \%$ and $19 \%$ of the response induced by TN2 in TMK1 (fig $1 \mathrm{~A}$ ) and MKN45 (fig 1B), respectively. SRE transactivation induced by $\mathrm{TN} 2-\Delta c a g E$ was also reduced compared with intact TN2 to $65 \%$ in TMK1 and $18 \%$ in MKN45. No transactivation was observed with heat killed TN2 or with

TMK1

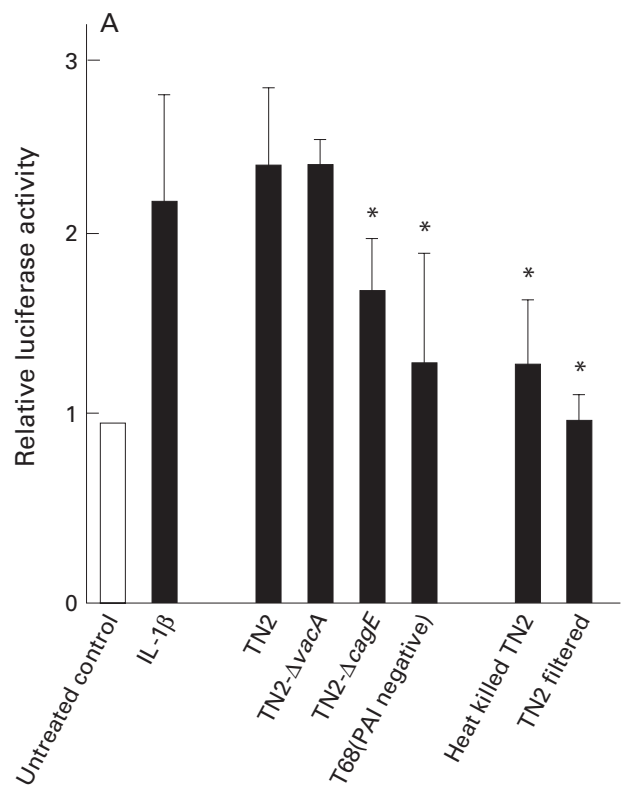

viable TN2 separated by a permeable membrane (fig 1A, B).

AP-1 TRANSACTIVATION BY $H$ PYLORI

Coculture with TN2 increased AP-1 transactivation in TMK1 and MKN45 to $240 \%$ and

Figure 2 Transactivation of AP-1 induced by Helicobacter pylori. Transactivation of AP-1 in TMK1 (A) and MKN45 (B) cells induced by $H$ pylori was measured by luciferase assay using pAP-1-Luc. The TN2 strain was positive for two known virulence factors, CagA and VacA, and had intact cag pathogenicity island (PAI). The T68 strain, isolated from a Fapanese patient at our institution, was negative for the above two virulence factors and lacked cag PAI. An isogenic cagE negative mutant (TN2- $\triangle$ cagE) was constructed by inserting a kanamycin resistant gene cassette into the cagE locus of cag PAI of TN2. The isogenic vacA negative mutant (TN2-4vacA) was constructed by disrupting the vacA gene of TN2. Luciferase activity is presented as a fold induction relative to basal levels measured in untreated cells. Mean (SD) values of four independent experiments are shown. ${ }^{\star} p<0.05$ compared with TN2 by Dunnett's multiple comparison. 


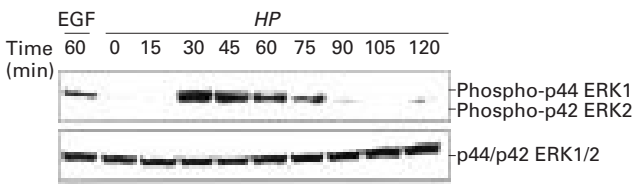

Figure 3 Phosphorylation of ERK1/2 by Helicobacter pylori $(H P)$. Immunoblot analysis showing phospho-ERK1/2 (top panel) and total ERK1/2 (bottom panel) in TMK1 cells cocultured with H pylori. Antibodies used react with both ERK1 and ERK2. Stimulation by epidermal growth factor ( $E G F 10 \mathrm{ng} / \mathrm{ml}$ for 60 minutes) was used as a positive control.

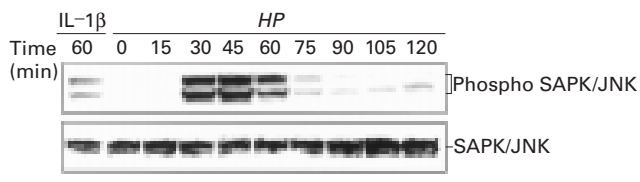

Figure 4 Phosphorylation of $7 N K / S A P K$ by Helicobacter pylori (HP). Immunoblot analysis showing phospho $7 N K$ (top panel) and total FNK (bottom panel) in TMK1 cells cocultured with $H$ pylori. Stimulation by interleukin $1 \beta$ (IL-1ß $10 \mathrm{ng} / \mathrm{ml}$ for 60 minutes) was used as a positive control.

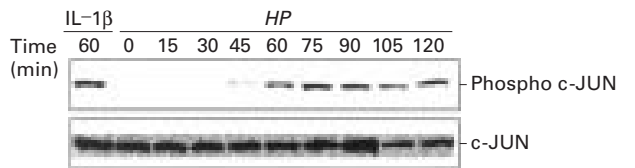

Figure 5 Phosphorylation of c-fun by Helicobacter pylori (HP). Immunoblot analysis showing phospho c-fUN (top panel) and total c-FUN (bottom panel) in TMK1 cells cocultured with $H$ pylori. Stimulation by interleukin $1 \beta$ (IL-1 $10 \mathrm{ng} / \mathrm{ml}$ for $60 \mathrm{~min}$ ) was used as a positive control.

$370 \%$ of responses in untreated cells, respectively (fig $2 \mathrm{~A}, 2 \mathrm{~B}$ ). While TN2- $\Delta$ vac $A$ induced $\mathrm{AP}-1$ transactivation comparable with $\mathrm{TN} 2$, AP-1 transactivation by $\mathrm{T} 68$ was reduced to $54 \%$ and $38 \%$ of responses induced by TN2 in TMK1 (fig 2A) and MKN45 (fig 2B), respectively. TN2- $\triangle c a g E$ induced an intermediate response in TMK1 $(71 \%)$ and a reduced response in MKN45 (38\%). No transactivation was induced with heat killed TN2 or with viable TN2 separated by a membrane filter.

PHOSPHORYLATION OF ERK1/2, JNK, AND c-JUN BY H PYLORI

The intracellular level of phosphorylated ERK1/2 was increased at 30 minutes in TMK1 cells cocultured with $H$ pylori (TN2) (fig 3). This phosphorylation was more prominent than that induced by EGF $(10 \mathrm{ng} / \mathrm{ml})$ and lasted for another 60 minutes. The intracellular level of total ERK1/2 remained constant. The level of phosphorylated JNK was increased at 30 minutes in TMK1 cells cocultured with TN2 (fig 4), and the level of phosphorylated c-Jun was increased in TMK1 cells after 60 minutes of co-culture with $H$ pylori (fig 5).

EFFECTS OF MEK INHIBITION

When TMK1 cells were pretreated with the MEK inhibitor U0126, $H$ pylori induced transactivation of SRE and AP-1 was significantly inhibited by $83 \%$ and $83 \%$, respectively (fig 6). The value of IL- 8 secretion from unstimulated cells was 1626 (435) pg/ml. The MEK inhibitor also inhibited $H$ pylori induced secretion of

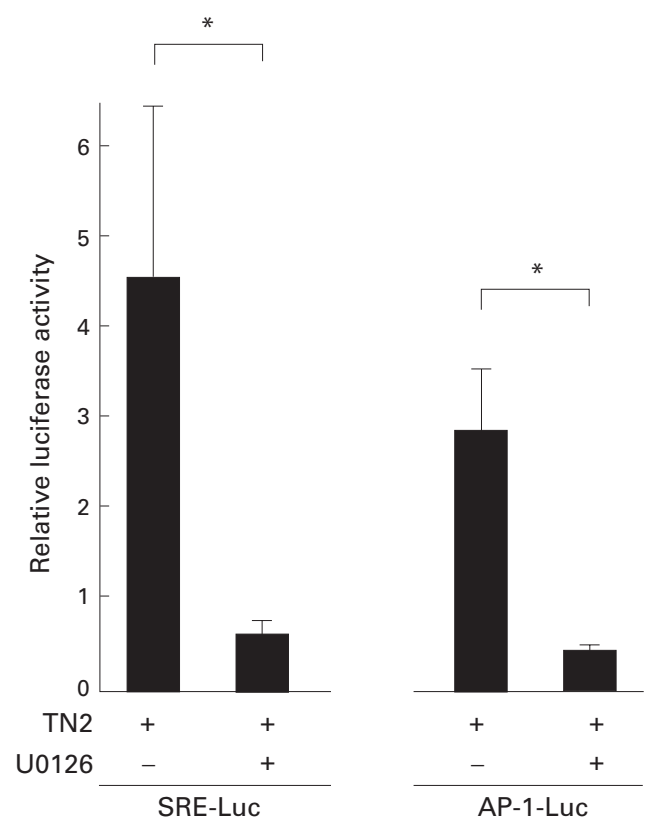

Figure 6 Effects of MEK inhibitor on Helicobacter pylori induced transactivation of $S R E$ and AP-1. Transactivation of SRE and AP-1 in TMK1 cells induced by $H$ pylori was measured by luciferase assay using pSRE-Luc and $p A P-1-L u c$ with $(+)$ or without $(-)$ the MEK inhibitor U0126 $(10 \mu \mathrm{M})$. Luciferase activity is presented as a fold induction relative to basal levels measured in untreated cells. Mean (SD) values of three independent experiments are shown. ${ }^{\star} p<0.05$ by unpaired Student's $t$ test.

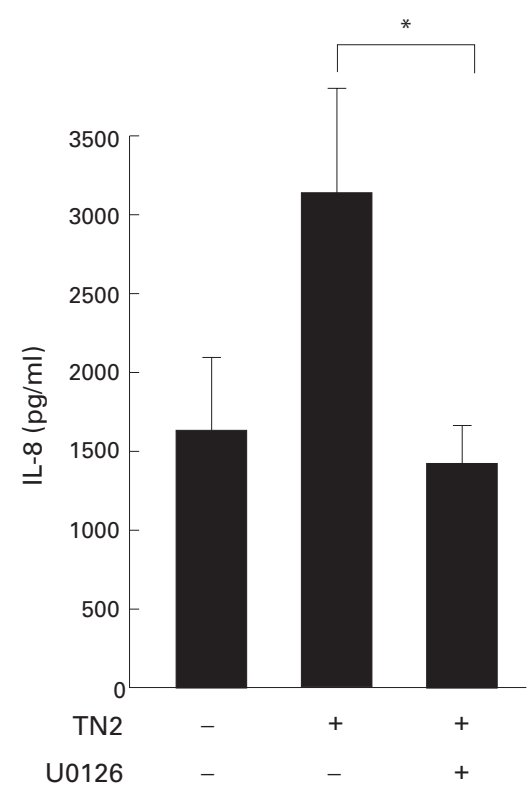

Figure 7 Effects of MEK inhibitor on Helicobacter pylori induced interleukin $8(I L-8)$ secretion. TMK1 cells were cultured alone as control or were cocultured with $H$ pylori with $(+)$ or without $(-)$ the MEK inhibitor U0126 (10 $\mu M)$. Supernatant IL-8 concentrations are shown. Mean $(S D)$ values of three independent experiments. ${ }^{*} p<0.05$ by unpaired Student's t test.

IL-8 from TMK1 cells by 53\% (from 3095 (666) to 1443 (221) $\mathrm{pg} / \mathrm{ml}$ ) (fig 7).

\section{Discussion}

We have confirmed in this study that SRE and AP-1 are transactivated in gastric cancer cells when cocultured with $H$ pylori. We also demonstrated not only phosphorylation of 
ERK1/2 in the ERK/MAPK cascade upstream of SRE but also phosphorylation of JNK and c-Jun in the JNK/SAPK cascade upstream of AP-1. Furthermore, the MEK inhibitor U0126 inhibited transactivation of not only SRE but also AP-1. Although there are no SRE sites in the promoter region of IL-8, induction of IL-8 secretion was inhibited by U0126. These results indicate that $H$ pylori infection transactivates AP-1 through activation of both the JNK/SAPK cascade, resulting in c-Jun activation, and the ERK/MAPK cascade, resulting in c-Fos expression. Although AP-1 can be transactivated by c-Jun homodimer, inhibition of AP-1 transactivation by MEK inhibitor indicates that the c-Jun/c-Fos heterodimer is the main form of AP-1 in $H$ pylori infection.

$H$ pylori infection in gastric mucosa induces not only IL- 8 but also IL- 6 and TNF- $\alpha$ whose promoter regions contain AP-1 binding sites. ${ }^{24-26}$ The TNF- $\alpha$ gene also has a NFKB binding site in the promoter region, and $H$ pylori infection is known to activate $\mathrm{NF \kappa B}$. Thus $H$ pylori infection may induce TNF- $\alpha$ through transactivation of both $\mathrm{AP}-1$ and $\mathrm{NF} \kappa \mathrm{B}$ binding sites, and $\mathrm{TNF}-\alpha$ binding to its receptors on adjacent cells will switch on intracellular signals leading to inflammatory and immunological responses.

Although the pathway upstream of the ERK/ MAPK cascade activated in $H$ pylori infection has not yet been identified, our study indicated that direct contact with viable $H$ pylori having intact cag PAI is required for the onset of signalling, resulting in induction of IL-8 secretion. Clinical isolates lacking cag PAI did not transactivate SRE or AP1 while those with intact cag PAI did (unpublished data).

During submission of this paper it was reported that $H$ pylori activates MAP kinase cascades and induces expression of $c-f o s$ and c-jun. ${ }^{27}$ However, enhancement of SRE, a key cis element of $c$-fos, was not shown directly. Furthermore, the importance of direct contact with viable $H$ pylori having intact cag PAI was not assessed.

These phenomena are difficult to interpret if molecules secreted by $H$ pylori or attached on the surface of bacterial body triggers the signals by binding unknown receptors. It is supposed that (some of) cag PAI genes construct a type IV secretion system that transports bacterial derived molecules into host cells. ${ }^{28}$ Thus the cascade may be triggered by some molecules of bacterial origin transported into host cells. Recently, one of the cag PAI products, CagA, was shown to be inserted into host cells ${ }^{29}$ although its relation to the cascades is not known.

In conclusion, we have confirmed transactivation of SRE and AP-1 through the ERK/ MAPK and JNK/SAPK cascades, respectively, in gastric cancer cells cocultured with $H$ pylori. Direct contact with viable bacteria possessing intact cag PAI is a prerequisite for the onset of intracellular signalling leading to AP-1 transactivation.
We thank Ms Mitsuko Tsubouchi for excellent technical assistance.

1 Warren JR, Marshall BJ. Unidentified curved bacilli on gasric epithelium in active chronic gastritis. Lancet $1983 ; 1: 1273-5$

2 Marshall BJ, Warren JR. Unidentified curved bacilli in the stomach of patients with gastritis and peptic ulceration. Lancet 1984;1:1311-15.

3 Censini S, Lange C, Xiang Z, et al. A. cag, a pathogenicity island of Helicobacter pylori, encodes type I-specific and disease-associated virulence factors. Proc Natl Acad Sci USA 1996;93:14648-53.

4 Crabtree JE, Farmery SM, Lindley IJ, et al. CagA/cytotoxic strains of Helicobacter pylori and interleukin-8 in gastric epithelial cell lines. F Clin Pathol 1994;47:945-50.

5 Huang J, O'Toole PW, Doig P, et al. Stimulation of interleukin-8 production in epithelial cell lines by Helicointerleukin-8 production in epithelial cell lines

6 Aihara M, Tsuchimoto D, Takizawa H, et al. Mechanisms involved in Helicobacter pylori-induced interleukin-8 production by a gastric cancer cell line, MKN45. Infect Immun duction by a gastric

7 Keates S, Hitti YS, Upton M, et al. Helicobacter pylori infec-

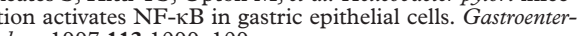
ology 1997;113:1099-109.

8 Glocker E, Lange C, Covacci A, et al. Proteins encoded by the cag pathogenicity island of Helicobacter pylori are required for NF-кB activation. Infect Immun 1998;66: 2346-8

9 Chauhan D, Kharbanda S, Uchiyama H, et al. Identification of upstream signals regulating interleukin- 6 gene expression during in vitro treatment of human $B$ cells with pokeweed mitogen. Blood 1994;84:2243-52.

10 Jain J, Valge-Archer VE, Rao A. Analysis of the AP-1 sites in the IL-2 promoter. F Immunol 1992;15:1240-50.

11 Rao A. NF-ATp: a transcription factor required for the co-ordinate induction of several cytokine genes. Immunol Today 1994;15:274-81.

12 Faisst S, Meyer S. Compilation of vertebrate-encoded transcription factors. Nucleic Acids Res 1992;20:3-26

13 Tsuji Y, Torti SV, Torti FM. Activation of the ferritin $\mathrm{H}$ enhancer, FER-1, by the cooperative action of members of the AP1 and Sp1 transcription factor families. F Biol Chem 1998;273:2984-92.

14 Angel P, Karin M. The role of Jun, Fos and the AP-1 complex in cell-proliferation and transformation. Biochim Biophys Acta 1991;1072:129-57.

15 Naumann M, Wessler S, Bartsch C, et al. Activation of activator protein 1 and stress response kinases in epithelial cells colonized by Helicobacter pylori encoding the cag pathogenicity island. F Biol Chem 1999;274:31655-62.

16 Hill CS, Wynne J, Treisman R. The Rho family GTPases RhoA, Rac1, and CDC42Hs regulate transcriptional activation by SRF. Cell 1995;81:1159-70.

17 Liao J, Hodge C, Meyer D, et al. Growth hormone regulates ternary complex factors and serum response factor associated with the c-fos serum response element. $\mathcal{F}$ Biol Chem ated with the c-fos

18 Maeda S, Yoshida H, Ikenoue T, et al. Structure of cag pathogenicity island in Japanese Helicobacter pylori isolates. Gut 1999;44:336-41.

19 Watanabe T, Tada M, Nagai $\mathrm{H}$, et al. Helicobacter pylori infection induces gastric cancer in Mongolian gerbils. Gastroenterology 1998;115:642-8.

20 Ogura K, Takahashi M, Maeda S, et al. Interleukin-8 production in primary cultures of human gastric epithelial cells induced Helicobacter pylori. Dig Dis Sci 1998;43:273843.

21 Maeda S, Ogura K, Yoshida $\mathrm{H}$, et al. Major virulence factors, VacA and CagA, are commonly positive in Helicobacter pylori isolates in Japan. Gut 1998;42:338-43.

22 Ochiai A, Yasui W, Tahara E. Growth-promoting effect of gastrin on human gastric carcinoma cell line TMK-1. fpn $\mathcal{F}$ Cancer Res 1985;76:1064-71.

23 Favata MF, Horiuchi KY, Manos EJ. Identification of a novel inhibitor of mitogen-activated protein kinase kinase. f Biol Chem 1998;273:18623-32.

24 Crabtree JE, Shallcross TM, Heatley RV, et al. Mucosal tumour necrosis factor alpha and interleukin- 6 in patients with Helicobacter pylori associated gastritis. Gut 1991;32: 1473-7.

25 Gionchetti P, Vaira D, Campieri M, et al. Enhanced mucosal interleukin-6 and -8 in Helicobacter pylori-positive dyspeptic patients. Am f Gastroenterol 1994;89:883-7.

26 Moss SF, Legon S, Davies J, et al. Cytokine gene expression in Helicobacter pylori associated antral gastritis. Gut 1994; 35:1567-70.

27 Meyer-ter-Vehn T, Covacci A, Kist M, et al. Helicobacter pylori activates mitogen-activated protein kinase cascades pylori activates mitogen-activated protein kinase cascades
and induces expression of the proto-oncogenes c-fos and and induces expression of the proto-on

28 Covacci A, Telford JL, Del Giudice G, et al. Helicobacter pylori virulence and genetic geography. Science 1999;284: 1328-33.

29 Segal ED, Cha J, Lo J, et al. Altered states: involvement of phosphorylated CagA in the induction of host cellular growth changes by Helicobacter pylori. Proc Natl Acad Sci USA 1999;96:14559-64. 